\title{
Hard X-ray induced fast secondary electron cascading processes in solids
}

K. Mecseki, H. Höppner, M. Büscher, V. Tkachenko, N. Medvedev, J. J. Bekx, V. Lipp, P. Piekarz, M. Windeler, J. W. G. Tisch, D. J. Walke, M. Nakatsutsumi, M. J. Prandolini, J. M. Glownia, T. Sato, M. Sikorski, M. Chollet, U. Teubner, J. Robinson, S. Toleikis, B. Ziaja, and F. Tavella

Citation: Appl. Phys. Lett. 113, 114102 (2018); doi: 10.1063/1.5046070

View online: https://doi.org/10.1063/1.5046070

View Table of Contents: http://aip.scitation.org/toc/apl/113/11

Published by the American Institute of Physics

\section{Conference Proceedings} print proceedings! 


\title{
Hard X-ray induced fast secondary electron cascading processes in solids
}

\author{
K. Mecseki, ${ }^{1, a)}$ H. Höppner, ${ }^{2,3,4}$ M. Büscher, ${ }^{3,4}$ V. Tkachenko, ${ }^{3,5}$ N. Medvedev, ${ }^{6,7}$ \\ J. J. Bekx, ${ }^{5,8}$ V. Lipp, ${ }^{5}$ P. Piekarz, ${ }^{9}$ M. Windeler, ${ }^{1,10}$ J. W. G. Tisch, ${ }^{11}$ D. J. Walke, ${ }^{11}$ \\ M. Nakatsutsumi, ${ }^{12}$ M. J. Prandolini, ${ }^{13}$ J. M. Glownia, ${ }^{1}$ T. Sato, ${ }^{1}$ M. Sikorski, ${ }^{1}$ M. Chollet, ${ }^{1}$ \\ U. Teubner, ${ }^{3,4}$ J. Robinson, ${ }^{1}$ S. Toleikis, ${ }^{14}$ B. Ziaja, ${ }^{5,9}$ and F. Tavella ${ }^{1}$ \\ ${ }^{1}$ SLAC National Accelerator Laboratory, Menlo Park, California 94025, USA \\ ${ }^{2}$ Institute for Radiation Physics, Helmholtz-Zentrum Dresden-Rossendorf e.V., 01328 Dresden, Germany \\ ${ }^{3}$ Institut für Laser und Optik, Hochschule Emden/Leer-University of Applied Sciences, 26723 Emden, Germany \\ ${ }^{4}$ Institut für Physik, Carl von Ossietzky Universität, 26111 Oldenburg, Germany \\ ${ }^{5}$ Center for Free-Electron Laser Science CFEL, Deutsches Elektronen-Synchrotron DESY, 22607 Hamburg, \\ Germany \\ ${ }^{6}$ Institute of Physics, Czech Academy of Sciences, Na Slovance 2, Prague 818221 , Czechia \\ ${ }^{7}$ Institute of Plasma Physics, Czech Academy of Sciences, Za Slovankou 3, Prague 8 18200, Czechia \\ ${ }^{8}$ Department of Physics, University of Hamburg, 20355 Hamburg, Germany \\ ${ }^{9}$ Institute of Nuclear Physics, Polish Academy of Sciences, Krakow 31-342, Poland \\ ${ }^{10}$ Queen's University, Kingston, Ontario K7L 3N6, Canada \\ ${ }^{11}$ Imperial College London, SW7 2 AZ London, United Kingdom \\ ${ }^{12}$ European XFEL, 22869 Schenefeld, Germany \\ ${ }^{13}$ Institute of Experimental Physics, University of Hamburg, 22761 Hamburg, Germany \\ ${ }^{14}$ Deutsches Elektronen-Synchrotron DESY, 22607 Hamburg, Germany
}

(Received 25 June 2018; accepted 30 August 2018; published online 14 September 2018)

\begin{abstract}
Recent studies confirmed that the materials used in the extreme UV and soft X-ray regime for precise characterization of intense free-electron laser pulses (e.g., $\mathrm{Si}_{3} \mathrm{~N}_{4}$ ) do not work efficiently in the hard X-ray regime, which is due to the fact that the impact of a hard X-ray photon is followed by a series of electron cascading processes. Following theoretical indication, we show that this limitation can be circumvented and the cascading time can be significantly reduced if the X-ray photon energy is double the ionization energy. We investigate an alternative material for pulse diagnostics, $\mathrm{SnO}_{2}$, using the Linac Coherent Light Source at photon energies of $5 \mathrm{keV}$ and $9 \mathrm{keV}$. We prove the validity of the concept and show that it has a large potential for practical applications. By applying the proposed criteria, the temporal accuracy of the non-invasive pulse diagnostic tools can be improved in current and emerging hard X-ray facilities. Published by AIP Publishing.

https://doi.org/10.1063/1.5046070
\end{abstract}

Free-electron lasers ${ }^{1-4}$ (FELs) deliver coherent, high peak brightness hard X-ray pulses with few to tens of femtosecond duration. These hard X-ray sources enable highresolution structural imaging, ${ }^{5}$ direct real time tracking of photo-induced dynamics in biological samples, ${ }^{6}$ and various physical $^{7}$ and chemical $^{8}$ processes. The majority of these experiments are based on pump-probe schemes, which allow the investigation of transient states of matter with a few femtosecond resolution. However, for the reconstruction of the entire dynamics of the studied system, the complete characterization of the pump and probe pulses with known delay is required. ${ }^{9}$

It was already observed during the first solid-state experiments with FELs that an intense FEL laser pulse excites many electrons within the irradiated solid. This leads to a transient change of the optical properties within the target that follows the evolution of the free-electron density. This behavior allows us to estimate precisely the delay between the pump and the probe pulse. ${ }^{10}$ Therefore, during the first $^{11,12}$ and later experiments ${ }^{13-15}$ measuring the transient optical properties of solids, the detected changes have been used to design non-destructive time-delay diagnostic ${ }^{9}$ and pulse characterization tools for FELs. ${ }^{16,17}$ For high resolution

a)kmecseki@slac.stanford.edu measurements, materials with a fast and strong electronic response to X-ray exposure are required.

As the interaction of the femtosecond hard X-ray pulse with materials leads to a sequence of complex processes, the interpretation of the measurement of the material's response requires a good understanding of the complex ionization dynamics. As mentioned above, by studying the transient change in the transmission of a sample, we can analyze the electron kinetics. The duration of the electron cascading process is dependent on the choice of the material and the X-ray photon energy. ${ }^{18}$ The material response at extreme ultraviolet (XUV) and soft X-ray wavelengths is nearly instantaneous. However, in the hard X-ray regime, photoabsorption and Auger decays release fast electrons from the core shells that further ionize upper shells. As a result, the pulse characterization technique mentioned above becomes challenging due to the effect of the Auger cascades and the following free electron cascading.

In this paper, we study the ultrafast electron cascading process upon hard X-ray irradiation of thin solid targets in a single shot. We demonstrate with high resolution measurements that materials with absorption characteristics tailored for a particular X-ray photon energy range can be used to increase the temporal resolution in X-ray arrival time and pulse duration monitoring diagnostics. We compare the 
measured responses for irradiation with $5 \mathrm{keV}$ and $9 \mathrm{keV}$ photon energies to the predicted response calculated from the theoretical model. ${ }^{18}$ According to Ref. 18, in some selected materials, where an ionization potential equals approximately half of the photon energy: $I_{p} \approx \hbar \omega / 2$, electron cascading will occur on a shorter timescale. In this case, the photon energy is shared between two electrons, a photo-electron and an Auger electron, each carrying about half of the photon energy. ${ }^{19}$ The optimal target material for X-ray arrival time and pulse duration measurements for a particular photon energy (i) has short electron cascading to achieve high temporal resolution, (ii) has high transparency and high damage threshold to minimize the impact on the transmitted X-ray pulses, (iii) while having a strong X-ray-induced optical modulation in order to maximize the measured optical signal.

The experiment was carried out at the Linac Coherent Light Source (LCLS) X-ray pump-probe (XPP) instrument. ${ }^{20}$ Considering reliable operation, $9 \mathrm{keV}$ was chosen as the highest photon energy for the measurements. Due to the relatively high atomic number of tin $(\mathrm{Z}=50), \mathrm{SnO}_{2}$ is an optimal diagnostic material at $9 \mathrm{keV}$ photon energy due to the fact that the L-shell ionization potential lies within the optimal range of half of the photon energy, $I_{p} \approx 3.9-4.4 \mathrm{keV} .{ }^{21}$ We show that this material exhibits inherently faster electron cascading processes at $9 \mathrm{keV}$, in comparison to the currently used hard $\mathrm{X}$-ray diagnostic materials, such as silicon nitride $\left(\mathrm{Si}_{3} \mathrm{~N}_{4}\right) \cdot \mathrm{Si}_{3} \mathrm{~N}_{4}$ is frequently used, because of its strong X-ray induced optical modulation and its availability.

We use an in-house Monte Carlo toolkit, XCASCADE ${ }^{18}$ for modeling the electron kinetics and the electron cascading processes occurring in solid targets. XCASCADE traces each electron and hole as individual particles with event-byevent simulation. It includes the photoabsorption by atoms, and the Auger decays of the produced core-holes, which result in the production of secondary energetic electrons and further electron impact ionizations. These processes lead to the distribution of the energy among an increasing number of electrons, until they can no longer make impact ionizations, at which point the cascading stops. The analog Monte Carlo scheme is used for modeling the electron kinetics. ${ }^{22}$ The atomic orbital energies are parametrized, using the atomic energy levels from the EADL-database. ${ }^{23}$ Photoabsorption cross sections for each atomic shell are taken from the EPDL97 database. ${ }^{24}$ The results were averaged over 10000 iterations to minimize statistical errors to $\sim 1 \%$. XCASCADE also predicted that less than $\sim 15 \%-20 \%$ of the absorbed energy could escape from both the front and the back surfaces of the irradiated films due to electron emission. Thus, this effect was neglected in the simulations.

In order to compare the response of $\mathrm{SnO}_{2}$ to $\mathrm{Si}_{3} \mathrm{~N}_{4}$, we perform a simulation study using the typical $\mathrm{X}$-ray pulse parameters available at the LCLS XPP instrument. The typical Self-Amplified Spontaneous Emission (SASE) X-ray pulses consist of a large number of coherent spikes of varying magnitudes from shot-to-shot. At present, the longitudinal properties of the pulses are estimated by indirect methods. In particular, at LCLS a transverse e-beam deflector (XTCAV) is used. ${ }^{25}$ In the simulations, the temporal duration of the X-ray pulses was assumed to be 25 fs fullwidth at half maximum (FWHM), which is close to values determined by the XTCAV (see supplementary material, Table S1). Additionally, the pulses were assumed to have a Gaussian temporal distribution, as the temporal distribution (envelope) of relatively long X-ray pulses with a large number of modes can be best fitted by a Gaussian shape. However, the presented method is not limited to pulses with a Gaussian temporal profile. The following X-ray fluences were determined from the measured $\mathrm{X}$-ray beam profiles and pulse energies (from a gas detector monitor) and applied in the simulations: $1.3,0.4 \mathrm{~J} / \mathrm{cm}^{2}$ at $5 \mathrm{keV}$ and $1.5,0.4 \mathrm{~J} / \mathrm{cm}^{2}$ at $9 \mathrm{keV}$ photon energies for $\mathrm{Si}_{3} \mathrm{~N}_{4}$ and $\mathrm{SnO}_{2}$, respectively. The evolution of the resulting $\mathrm{X}$-ray induced electron density change occurs on the timescale of 50-100 fs. Figure 1 shows a comparison of the results (a) and (c) highlighting the derivatives of the electron densities to better illustrate the duration of the processes (b) and (d).

The model shows that both test materials have similar responses at $5 \mathrm{keV}$, with a marginally faster response for $\mathrm{Si}_{3} \mathrm{~N}_{4}$ when compared to that of $\mathrm{SnO}_{2}$. At $9 \mathrm{keV}$, the increase in the electron density for $\mathrm{SnO}_{2}$ is significantly faster. Additionally, the maximum of the density reaches a value more than one order of magnitude higher than in $\mathrm{Si}_{3} \mathrm{~N}_{4}$, providing a better signal to noise ratio. Figures 1(e) and 1(f) illustrates the calculated FWHM of the electron density derivatives for both materials at different photon energies. When the photon energy is in the optimal range for the material, the timescale of the response reflects more accurately the FEL pulse duration, as the electron cascading process is faster and influences the response time less.

The measurements of the cascading processes were carried out both at $5 \mathrm{keV}$ and $9 \mathrm{keV} \mathrm{X-ray} \mathrm{photon} \mathrm{energies.} \mathrm{The}$ experimental setup is based on X-ray/optical cross-correlation. ${ }^{11,16,17}$ The measurement of the transient change of the complex refractive index within the irradiated material was carried out via a transmission measurement in a thin target material.

A schematic of the experimental setup is shown in Fig. 2. The thin substrate is mounted on a motorized XY stage. The unfocused X-ray beam is partially absorbed in a solid sample, incident at an angle of $30^{\circ}$ with respect to the target normal. This choice of incidence angle yields an adequate time window with high resolution (206.9 fs time window with a 0.2011 fs/pixel resolution), while keeping the geometrical smearing effects at a minimal level. Smearing of the signal occurs due to the in depth absorption of the FEL beam in the sample and quantifies at an angle of $\theta$ as: $n T / c \sin \theta$, where $n$ is the refractive index of the material in the visible regime, $T$ is the thickness of the interaction length, and $c$ is the speed of light in vacuum. This manifests itself as a $\sim 2$ fs broadening of the signal when using a sample with a thickness of $\sim 0.5 \mu \mathrm{m}$. The thicknesses of the $\mathrm{SnO}_{2}$ and $\mathrm{Si}_{3} \mathrm{~N}_{4}$ samples are chosen to be $487.3 \pm 27 \mathrm{~nm}$ and $563.5 \pm 2.5 \mathrm{~nm}$, respectively, to minimize smearing effects. The sample thicknesses are measured with ellipsometry (HORIBA UVISEL FUV-VIS-IR 210-880 nm ellipsometer). The temporal evolution of the ionization process and the subsequent electron cascading processes are monitored with a probe laser with a wavefront parallel to the target. The probe pulses are prepared in a Mach-Zehnder-interferometer, where one interferometer arm is delayed by $1 \mathrm{ps}$, and the polarization is rotated orthogonally with respect to the polarization 

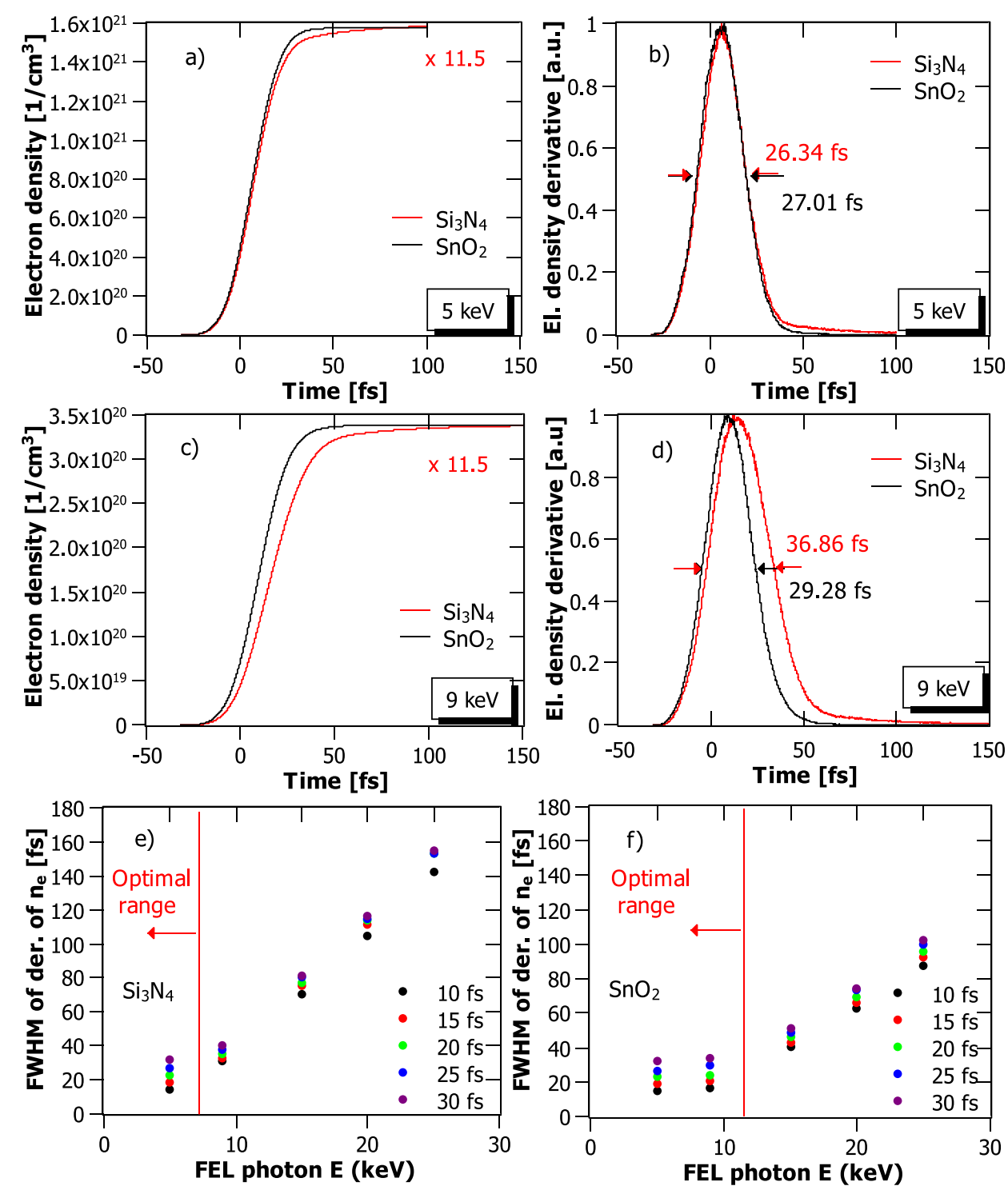

FIG. 1. Calculated evolution of the electron density (a) and (c), and time derivative of the electron density (b) and (d) for $\mathrm{Si}_{3} \mathrm{~N}_{4}$ and $\mathrm{SnO}_{2}$ materials, when irradiated with a 25 fs laser pulse at $5 \mathrm{keV}$ (a) and (b) and $9 \mathrm{keV}$ (c) and (d). The electron density function for $\mathrm{Si}_{3} \mathrm{~N}_{4}$ was multiplied by 11.5 on both (a) and (c) for easier comparison. The FWHM of the time derivative of the electron density is plotted to show the optimal range of photon energies where the electron cascading effects happen on a shorter timescale for (e) $\mathrm{Si}_{3} \mathrm{~N}_{4}$ (f) and $\mathrm{SnO}_{2}$, at different photon energies and FEL pulse durations. of the pulses in the other arm. The first laser pulse temporally overlaps with the X-ray pulse, in order to probe the progressing ionization process. The second pulse probes the sample after the X-ray pulse has passed through the material. The recorded image can be used as a calibration/reference image to eliminate any measurement error due to structural inhomogeneity of the FEL pulse or the target material. The X-ray pulse repetition rate in the measurements is set to $60 \mathrm{~Hz}$, while the repetition of the optical laser is $120 \mathrm{~Hz}$. This allows the recording of a consecutive, "laser only" background shot for each measurement, improving the quality of the measurement. The transmission is measured by imaging the illuminated target surface with a Mitutoyo $\times 20$ microscope objective onto two CCD cameras (Opal-1000, 10-bit) separated by a broadband cube polarizer (data/calibration). The collimated optical probe laser pulse has a parallel wavefront to the target surface.

The optical probe laser pulse was spectrally broadened in a gas-filled capillary and subsequently compressed with a chirped mirror setup. ${ }^{26}$ The pulse duration was measured with a single-shot frequency-resolved optical gating (FROG) to be $9.1 \pm 0.14$ fs measured at FWHM (see Fig. 2). The optical pulse was centered at $800 \mathrm{~nm}$ and had a pulse energy of $\sim 20$ $\mu \mathrm{J}$ on the target. We used a collimated FEL beam with a spot size on a target of $\sim 700 \times 200 \mu \mathrm{m}^{2}$, with a larger beam dimension along the time projection axis. The measured cross-correlation data can be used as an arrival-time monitoring tool of the X-ray and optical pulse. In order to measure the accuracy of our technique, during the measurements, the relative time of arrival between the FEL pulse and the optical probe pulse, and the FEL pulse duration was monitored using the XPP time tool ${ }^{27,28}$ and the XTCAV, ${ }^{25}$ respectively.

The measured temporal evolution of the transmission, triggered by the X-ray induced electron kinetics, reflects the electron cascading processes. The background corrected images (see "Transmission" recording in Fig. 2) are summed up along the spatial axis. The derivative of the measured transmission signal for $\mathrm{SnO}_{2}$ at $5 \mathrm{keV}$ is shown in Fig. 3(a). The laser pulse duration has been deconvolved in the time domain from each measurement, in order to obtain the intrinsic material response to the FEL pulse, and then the resulting profiles are normalized. The measurements are sorted by the FEL pulse arrival time calculated from our measured data. The measured arrival times are also correlated with the standard XPP time arrival monitor [Fig. 3(b)]. Figure 3(c) shows a histogram of the measurement with an accuracy of $\sigma=5.2 \mathrm{fs}$. The XPP time tool has an accuracy of $\sigma \sim 5-6$ fs r.m.s. ${ }^{9}$ The 
a)

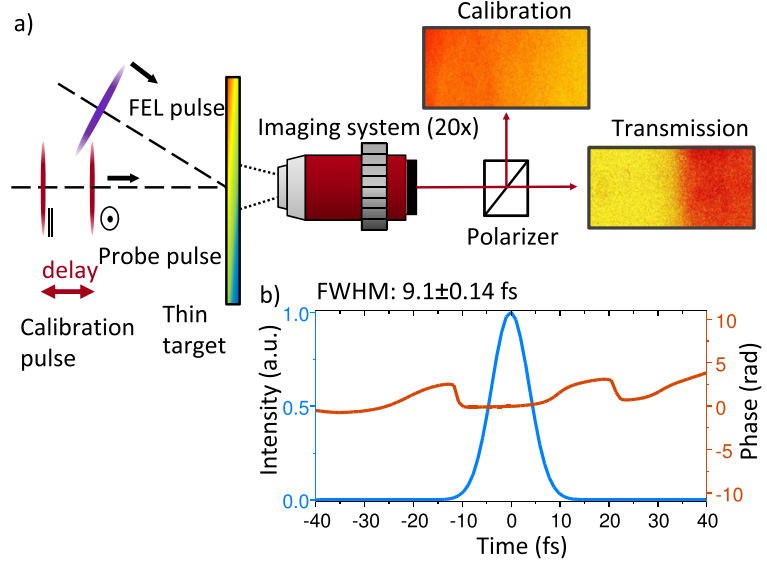

FIG. 2. (a) Schematic of the experimental setup for single-shot measurement of the electron cascading processes. The FEL pulse arriving at an angle of $30^{\circ}$ with respect to the target normal causes a fast ionization process, which is probed by an ultrashort $(<10 \mathrm{fs})$ long laser pulse at $800 \mathrm{~nm}$ wavelength at normal incidence. Different fractions of the X-ray wavefront are absorbed at different spatial positions on the sample, resulting in a spatio-temporal encoding of the ionization process. The first optical pulse probes the ionization process and then the outcome is recorded in the transmission measurement image. The surface of the thin target is imaged onto two CCD cameras separated by a broadband polarizer cube. (b) Reconstructed temporal intensity and phase profile of the probe pulse from a single shot FROG trace, $\mathrm{FWHM}=9.1 \pm 0.14$ fs.

measured standard deviation of the timing jitter between the $\mathrm{X}$-ray and the laser pulses during the experiment was on the order of $\sim 100-200 \mathrm{fs}$.

After calculating the derivative of the transmission, we performed the direct time domain deconvolution of the laser pulse duration to obtain the intrinsic material response to the FEL pulse. Figure 4 shows the response times (derivatives of the transmission) for both $\mathrm{SnO}_{2}$ and $\mathrm{Si}_{3} \mathrm{~N}_{4}$ at $5 \mathrm{keV}$ (a) and $9 \mathrm{keV}$ (b). The measurements are in very good agreement with the modeled transmission curves, also shown in Fig. 4. These curves were fitted with a model combining rate equations with the Drude model (for more details see supplementary material). This approach yields the transient values of the complex refractive index, which are then used to calculate the optical probe pulse transmission. The curves for $\mathrm{Si}_{3} \mathrm{~N}_{4}$ and $\mathrm{SnO}_{2}$ have similar characteristics at lower photon
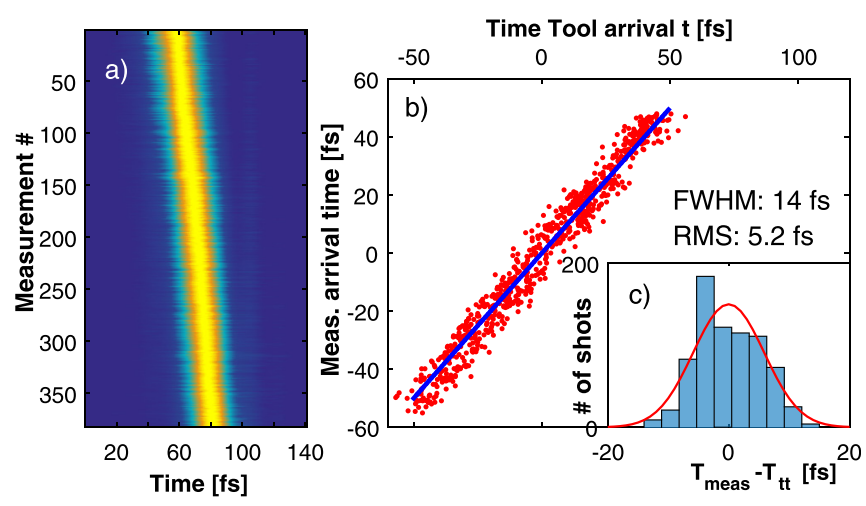

FIG. 3. (a) Derivative of the transmission of $\mathrm{SnO}_{2}$ at $5 \mathrm{keV}$. The data has been sorted by the FEL arrival time using our measurement technique: (b) correlation between standard XPP TimeTool diagnostics and our measured arrival time (red measurement points). The blue solid line $(\mathrm{x}=\mathrm{y})$ is a guide for the eye. (c) Histogram of the arrival time measurement differences shown for accuracy comparison.
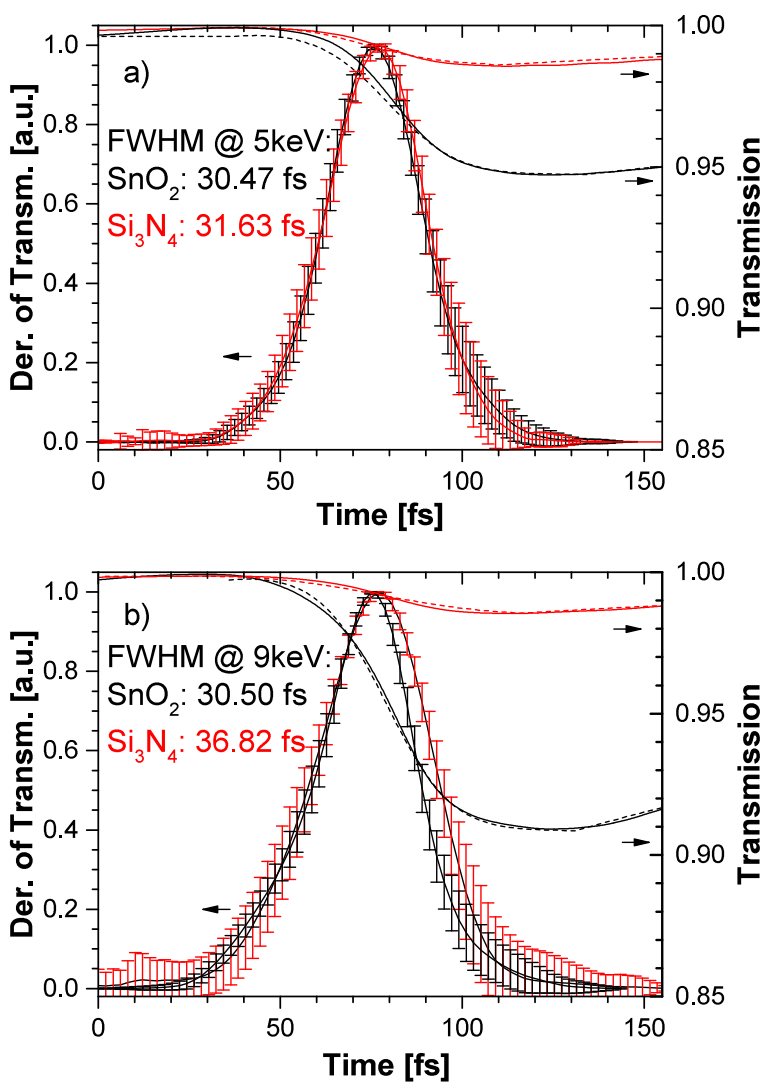

FIG. 4. (a) Measured (solid lines) and calculated (dashed lines) transmission (right axis) and the derivative of the measured transmission (left axis) of $\mathrm{SnO}_{2}$ (a) and (b) black, $\mathrm{Si}_{3} \mathrm{~N}_{4}$ (a) and (b) red at photon energies (a) $5 \mathrm{keV}$ and (b) $9 \mathrm{keV}$.

energy $(5 \mathrm{keV})$, while $\mathrm{SnO}_{2}$ exhibits a faster electron cascading time at higher photon energy $(9 \mathrm{keV})$. This is expected due to the ionization potential of $\mathrm{SnO}_{2}$ at $9 \mathrm{keV}$ being approximately half of the photon energy. In contrast, the duration of the electron cascading in $\mathrm{Si}_{3} \mathrm{~N}_{4}$ increases substantially (see Fig. 4), as it was predicted by the simulations. ${ }^{18} \mathrm{SnO}_{2}$, the material under consideration has a similar response to hard X-ray irradiation over a broad photon energy range. XCASCADE simulations show similar behavior at 5, 7 (not shown), and $9 \mathrm{keV}$ [Fig. 1(f)]. The measurements with $\mathrm{SnO}_{2}$ are performed with an FEL pulse duration of $24.45 \pm 5.75 \mathrm{fs}$ and $23.21 \pm 4$ fs at 5 and $9 \mathrm{keV}$, respectively. The measured and calculated FWHM of the transmission derivatives are $30.47 \pm 3.18 \mathrm{fs}$ and $32.67 \mathrm{fs}$ at $5 \mathrm{keV}$. The measurement and calculated values at $9 \mathrm{keV}$ are $30.50 \pm 2.63 \mathrm{fs}$ and $31.07 \mathrm{fs}$.

At present, a large number of scientific studies with free-electron lasers are performed using ultrafast optical/ $\mathrm{X}$-ray pump-probe techniques. These techniques require precise temporal X-ray diagnostics. Here, we presented the first high resolution single-shot measurements of the X-ray induced electron cascading effects in selected materials used for X-ray arrival time and pulse duration diagnostics. We intended to identify optimal diagnostic materials at high photon energies (e.g., $>5 \mathrm{keV}$ ) with short electron cascading times and high free-electron density produced. The measurement was inspired by the theoretical indication that within some selected materials for which the ionization energy of an atomic shell level is about half of the X-ray photon energy, the electron cascading 
can be significantly faster and thus, it does not perturb the measurement of the temporal pulse characteristics. Additionally, we present our measurement of the duration of the electron cascading in a currently widely used material, $\mathrm{Si}_{3} \mathrm{~N}_{4}$ at both 5 $\mathrm{keV}$ and $9 \mathrm{keV}$ photon energies. The results of $\mathrm{Si}_{3} \mathrm{~N}_{4}$ are compared to a proposed material with optimal ionization potential at $9 \mathrm{keV}, \mathrm{SnO}_{2}$. In this material, binding energies of the primary electrons initiating electron cascades approach half of the $9 \mathrm{keV}$ photon energy, which significantly reduces the cascading time compared to $\mathrm{Si}_{3} \mathrm{~N}_{4}$. Additionally, the observed transmission change in $\mathrm{SnO}_{2}$ is larger due to higher free-electron densities induced, even at lower X-ray pump energies, making this material potentially interesting for X-ray beam monitoring. The excellent agreement between measurements and theoretical simulation deepens our understanding of material response in the hard X-ray regime. The simple experimental methodology presented lays the foundation for single-shot pulse duration measurements in this spectral range.

See supplementary material for the theoretical framework used to describe the evolution of the electron distribution and the optical transmission as a function of time.

U. Teubner, V. Tkachenko, and M. Büscher acknowledge funding by the German Federal Ministry of Education and Research BMBF, Funding Code No. 05K16ME1. Partial financial support from the Czech Ministry of Education (Grant Nos. LG15013 and LM2015083) is acknowledged by N. Medvedev. D. J. Walke acknowledges funding support from the EPSRC (Grant No. EP/K503733/1).

${ }^{1}$ P. Emma, R. Akre, J. Arthur, R. Bionta, C. Bostedt, J. Bozek, A. Brachmann, P. Bucksbaum, R. Coffee, F.-J. Decker et al., Nat. Photonics 4, 641 (2010).

${ }^{2}$ D. Pile, Nat. Photonics 5, 456 (2011).

${ }^{3}$ R. Ganter, "Swissfel-conceptual design report," PSI Report 10-04 (Paul Scherrer Institute (PSI), 2010).

${ }^{4}$ M. Altarelli, Nucl. Instrum. Methods Phys. Res., Sect. B 269, 2845 (2011).

${ }^{5}$ H. N. Chapman, A. Barty, M. J. Bogan, S. Boutet, M. Frank, S. P. HauRiege, S. Marchesini, B. W. Woods, S. Bajt, W. H. Benner et al., Nat. Phys. 2, 839 (2006).

${ }^{6}$ A. Hosseinizadeh, G. Mashayekhi, J. Copperman, P. Schwander, A. Dashti, R. Sepehr, R. Fung, M. Schmidt, C. H. Yoon, B. G. Hogue, G. J. Williams, A. Aquila, and A. Ourmazd, Nat. Methods 14, 877 (2017).

${ }^{7}$ H. T. Lemke, K. S. Kjær, R. Hartsock, T. B. Van Driel, M. Chollet, J. M. Glownia, S. Song, D. Zhu, E. Pace, S. F. Matar et al., Nat. Commun. 8, 15342 (2017).
${ }^{8}$ M. W. Mara, R. G. Hadt, M. E. Reinhard, T. Kroll, H. Lim, R. W. Hartsock, R. Alonso-Mori, M. Chollet, J. M. Glownia, S. Nelson et al., Science 356, 1276 (2017).

${ }^{9}$ M. Harmand, R. Coffee, M. R. Bionta, M. Chollet, D. French, D. Zhu, D. Fritz, H. Lemke, N. Medvedev, B. Ziaja et al., Nat. Photonics 7, 215 (2013).

${ }^{10}$ U. Teubner, U. Wagner, and E. Förster, J. Phys. B: At., Mol. Opt. Phys. 34, 2993 (2001).

${ }^{11}$ C. Gahl, A. Azima, M. Beye, M. Deppe, K. Döbrich, U. Hasslinger, F. Hennies, A. Melnikov, M. Nagasono, A. Pietzsch et al., Nat. Photonics 2, 165 (2008).

${ }^{12}$ T. Maltezopoulos, S. Cunovic, M. Wieland, M. Beye, A. Azima, H. Redlin, M. Krikunova, R. Kalms, U. Frühling, F. Budzyn et al., New J. Phys. 10, 033026 (2008).

${ }^{13}$ M. Krikunova, T. Maltezopoulos, A. Azima, M. Schlie, U. Frühling, H. Redlin, R. Kalms, S. Cunovic, N. M. Kabachnik, M. Wieland et al., New J. Phys. 11, 123019 (2009).

${ }^{14}$ M. Krikunova, T. Maltezopoulos, P. Wessels, M. Schlie, A. Azima, M. Wieland, and M. Drescher, J. Chem. Phys. 134, 024313 (2011).

${ }^{15}$ M. Krikunova, T. Maltezopoulos, P. Wessels, M. Schlie, A. Azima, T. Gaumnitz, T. Gebert, M. Wieland, and M. Drescher, Phys. Rev. A 86, 043430 (2012).

${ }^{16}$ R. Riedel, A. Al-Shemmary, M. Gensch, T. Golz, M. Harmand, N. Medvedev, M. Prandolini, K. Sokolowski-Tinten, S. Toleikis, U. Wegner et al., Nat. Commun. 4, 1731 (2013).

${ }^{17}$ P. Finetti, H. Höppner, E. Allaria, C. Callegari, F. Capotondi, P. Cinquegrana, M. Coreno, R. Cucini, M. B. Danailov, A. Demidovich et al., Phys. Rev. X 7, 021043 (2017).

${ }^{18}$ N. Medvedev, Appl. Phys. B 118, 417 (2015).

${ }^{19}$ N. Medvedev, B. Ziaja, M. Cammarata, M. Harmand, and S. Toleikis, Contrib. Plasma Phys. 53, 347 (2013).

${ }^{20}$ M. Chollet, R. Alonso-Mori, M. Cammarata, D. Damiani, J. Defever, J. T. Delor, Y. Feng, J. M. Glownia, J. B. Langton, S. Nelson et al., J. Synchrotron Radiat. 22, 503 (2015).

${ }^{21}$ C. Tang, Z. Luo, Z. An, F. He, X. Peng, and X. Long, Phys. Rev. A 65, 052707 (2002).

${ }^{22}$ K. Nishimura, J. Kawata, and K. Ohya, Nucl. Instrum. Methods Phys. Res., Sect. B 164-165, 903 (2000).

${ }^{23}$ S. Perkins, D. Cullen, M. Chen, J. Rathkopf, J. Scofield, and J. Hubbell, "Tables and graphs of atomic subshell and relaxation data derived from the LLNL evaluated atomic data library (EADL), $z=1-100$," Technical Report No. UCRL-50400-Vol. 30 (Lawrence Livermore National Lab., CA, United States, 1991).

${ }^{24}$ D. E. Cullen, J. H. Hubbell, and L. Kissel, Report No. UCRL-50400 (1997), Vol. 6, p. 1.

${ }^{25}$ C. Behrens, F.-J. Decker, Y. Ding, V. Dolgashev, J. Frisch, Z. Huang, P. Krejcik, H. Loos, A. Lutman, T. Maxwell et al., Nat. Commun. 5, 3762 (2014).

${ }^{26}$ J. Robinson, C. Haworth, H. Teng, R. Smith, J. Marangos, and J. Tisch, Appl. Phys. B: Lasers Opt. 85, 525 (2006).

${ }^{27}$ S. Schorb, T. Gorkhover, J. P. Cryan, J. M. Glownia, M. Bionta, R. N. Coffee, B. Erk, R. Boll, C. Schmidt, D. Rolles et al., Appl. Phys. Lett. 100, 121107 (2012).

${ }^{28}$ M. Beye, O. Krupin, G. Hays, A. Reid, D. Rupp, S. d Jong, S. Lee, W.-S. Lee, Y.-D. Chuang, R. Coffee et al., Appl. Phys. Lett. 100, 121108 (2012). 